\title{
Terrestrial photogrammetry: a method to gather data on fractures for DFN modelling from exposed rock surfaces
}

\author{
Filip Loeckle \\ Bundesanstalt für Geowissenschaften und Rohstoffe (BGR), Hannover, 30655, Germany \\ Correspondence: Filip Loeckle (filip.loeckle@bgr.de)
}

Published: 10 November 2021

\begin{abstract}
The stochastic generation of discrete fracture networks (DFN) is a method for modelling fracture patterns used to assess the in situ fragmentation in a volume of rock. The DFN modelling approach is based on the assumption that the natural fragmentation of rocks is a function of the length and connectivity of the fractures within the considered volume of rock. Thus, in order to generate a site-specific DFN, the primary geometric properties of the fracture surfaces within the rock volume (especially orientation, size and fracture intensity as well as the local spatial variability) must be defined as distribution functions (Elmo et al., 2014). The required base statistics are usually obtained from fracture analysis on boreholes, exposed rock surfaces or (to a limited extent) 3D seismics (e.g. Bisdom et al., 2014; Bemis et al., 2014).

We adopted a terrestrial close-range photogrammetry approach to capture several outcrops and analyse fracture traces on the exposed rock surfaces, the chosen workflow is based around the use of free and open-source software. Images were acquired from several quarries in the Weschnitzpluton, a granodioritic to quartz monzodioritic pluton in the Bergstrasse Odenwald (e.g. Altherr et al., 1999) using a consumer-grade Nikon D5300 DSLR with fixed focal length instead of a drone or Lidar-system for legal reasons, partially tree-lined outcrops and cost efficiency. Since point clouds obtained from photogrammetry are inherently dimensionless, we used a spherical target with compass and bubble level for scale and proper spatial orientation (Froideval et al., 2019). The exact geolocation is not particularly important for the task, so the use of GPS, total station or georeferenced ground control points is not necessary. Dense point clouds were computed using the open source SfM photogrammetry suite Meshroom (AliceVision, 2021), which can be used for manual or semi-automatic detection of fracture surfaces and their orientation (Schnabel et al., 2007) and to generate orthorectified images of the rock surface to trace fracture lengths and nodes in a GIS (Nyberg et al., 2018). Our investigations proved terrestrial photogrammetry to be a valuable and easily accessible tool in the documentation of natural fracture patterns and a robust base for the generation of DFN networks.
\end{abstract}

Kurzfassung. Die stochastische Generierung diskreter Kluftnetzwerke (engl. discrete fracture networks, DFN) ist eine Methode zur Modellierung von Bruchmustern, die zur Abschätzung der in-situ Zerblockung natürlicher Gesteine verwendet wird. Der DFN-Ansatz basiert auf der Annahme, dass die Fragmentierung natürlicher Gesteine eine Funktion der Länge und Konnektivität der in dem betrachteten Gesteinsvolumen enthaltenen Brüche darstellt. Um ein standortspezifisches DFN zu generieren, müssen die primären geometrischen Eigenschaften der Bruchflächen in dem betrachteten Gesteinsvolumen als Verteilungsfunktionen definiert werden, insbesondere müssen hierfür Informationen zur Orientierung, Ausdehnung und Bruchintensität vorliegen, sowie deren räumliche Variabilität beschrieben werden (Elmo et al., 2014). Die dafür erforderlichen statistischen Datensätze werden üblicherweise aus Bohrlöchern oder Oberflächenaufschlüssen sowie eingeschränkt auch aus der 3D-Seismik gewonnen (z. B. Bisdom et al., 2014; Bemis et al., 2014).

Die Erfassung der Aufschlusswände erfolgte mittels Methodiken aus dem Bereich der terrestrischen Nahbereichsphotogrammetrie, für die Bruchstrukturanalyse wurde ausschliesslich freie und quelloffene Software verwendet. Die aufgenommenen Steinbrüche befinden sich im Weschnitzpluton, einem granodioritischen bis quarz-monzodioritischen Pluton im Bergsträßer Odenwald (z. B. Altherr et al., 1999). Für die photographische 
Aufnahme wurde eine handelsübliche Spiegelreflexkamera (Nikon D5300) mit Festbrennweitenobjektiv verwendet, Drohnen oder Lidar-Systeme wurden aus rechtlichen Gründen, wegen des teilweise vorhandenen Baumbestandes und aus Gründen der Kosteneffizienz nicht eingesetzt. Da durch photogrammetrische Methoden erzeugte Punktwolken grundsätzlich dimensionslos sind, wurde ein sphärisches Ziel mit Kompass und Libelle verwendet, um die erzeugten Modelle nachträglich skalieren und in ihre tatsächliche räumliche Lage rückorientieren zu können (Froideval et al., 2019). Die exakte globale Position ist für den Einsatzzweck nicht relevant, daher kann auf GPS, georeferenzierte Bodenkontrollpunkte oder Tachymeter ebenfalls verzichtet werden. Dichte dreidimensionale Punktwolkenmodelle wurden mittels des freien SfM-Photogrammetrie Softwarepakets Meshroom (AliceVision, 2021) erzeugt. Die Punktwolkenmodelle können anschliessend zur manuellen oder halbautomatischen Erfassung der Bruchflächenorientierungen (Schnabel et al., 2007) sowie für die Erzeugung von Orthofotos zur Digitalisierung von Bruchmustern und Analyse der Knotenpunkte mittels GIS (Nyberg et al., 2018) verwendet werden. Unsere Untersuchungen zeigen auf, dass die terrestrische Nachbereichsphotogrammetrie ein nützliches und leicht zugängliches Werkzeug zur Erfassung von Bruchmustern in Aufschlüsen darstellt, das eine robuste Datengrundlage für die stochastische Generierung von Kluftnetzwerken liefert.

\section{References}

AliceVision: Alice Vision Meshroom: An open-source 3D reconstruction pipeline, in: Proc. 12th ACM Multimed. Syst. Conf. - MMSys '21, 28 September-1 October 2021, Istanbul, Turkey https://doi.org/10.1145/3458305.3478443, 2021.

Altherr, R., Henes-Klaiber, U., Hegner, E., Satir, M., and Langer, C.: Plutonism in the Variscan Odenwald (Germany): from subduction to collision, Int. J. Earth Sci., 88, 422-443, https://doi.org/10.1007/s005310050276, 1999.

Bemis, S. P., Micklethwaite, S., Turner, D., James, M. R., Akciz, S., Thiele, S. T., and Bangash, H. A.: Ground-based and UAV-Based photogrammetry: A multi-scale, high-resolution mapping tool for structural geology and paleoseismology, J. Struct. Geol., 69, 163-178, https://doi.org/10.1016/j.jsg.2014.10.007, 2014.

Bisdom, K., Gauthier, B. D. M., Bertotti, G., and Hardebol, N. J.: Calibrating discrete fracture-network models with a carbonate three-dimensional outcrop fracture network: Implications for naturally fractured reservoir modeling, Am. Assoc. Petr. Geol. B., 98, 1351-1376, https://doi.org/10.1306/02031413060, 2014.
Elmo, D., Rogers, S., Stead, D., and Eberhardt, E.: Discrete Fracture Network approach to characterise rock mass fragmentation and implications for geomechanical upscaling, Mining Technology, 123, 149-161, https://doi.org/10.1179/1743286314Y.0000000064, 2014.

Froidevedal, L., Pedoja, K., Garester, F., Moulin, P., Conessa, C., Pellerin Le Bas, X., Traoré, K., and Benoit, L.: A lowcost open-source workflow to generate 3D photogrammetric models of rocky outcrops, Photogramm. Rec., 34, 365-384, https://doi.org/10.1111/phor.12297, 2019.

Nyberg, B., Nixon, C. W., and Sanderson, D. J.: NetworkGT: A GIS tool for geometric and topological analyses of twodimensional fracture networks, Geosphere, 14, 1618-1634, https://doi.org/10.1130/GES01595.1, 2018.

Schnabel, R., Wahl, R., and Klein, R.: Efficient RANSAC for PointCloud Shape Detection, Comput. Graph. Forum, 26, 214-226, 2007. 